\title{
Psychasthenia 3: Dupes
}

\author{
Joyce Rudinsky \\ University of North Carolina, $\mathrm{CH}$ \\ 110 Bingham Hall \\ Campus Box 3285 \\ Chapel Hill, NC 27599, USA \\ rudinsky@unc.edu
}

\author{
Victoria Szabo \\ Duke University \\ 114 S. Buchanan Blvd \\ Box 90766 \\ Durham NC 27701, USA \\ ves4@duke.edu
}

\section{INTRODUCTION}

Pyschasthenia 3: Dupes explores the use of the immersive 3D game environment Unity3D as a site for interactive new media artwork. This project is a product of the Psychasthenia Studio collaborative. As a game-based immersive experience, Dupes explores challenging workplace relationships and gamified assessment environments, revealing the ubiquity of data shadow construction, the erosion of personal privacy, and the extent to which the avatar self can substitute for the real person in contemporary workplace culture.

\section{GAMEPLAY}

The game is set in a dystopic, yet banal, workplace environment, where every interaction, whether "in person" or online, is logged and judged against a series of internal evaluation factors. The game culminates in the revelation that everything you have done as a user has been subject to tracking and interpretation.

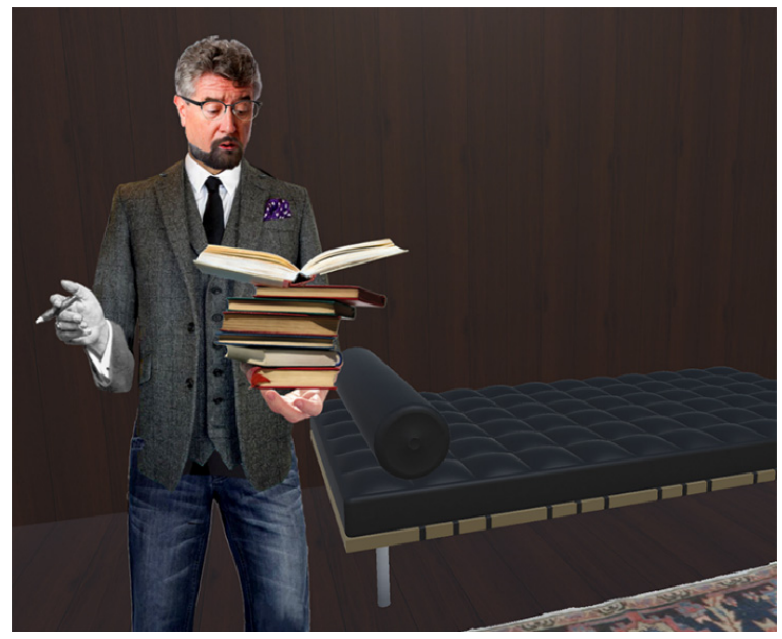

Figure 1: Consulting the Company Doctor

The user's in-world experience begins at an office desk, at 8am, and with a required HR test, which you are required to complete by close of business. The test questions you encounter are modified versions of the popular Big 5 personality test, which is currently in fashion as a measure of potential workplace success. This required test-taking, however, is interrupted repeatedly throughout the work day.

Conflicting demands are delivered via computer messages - a visit to the company shrink, to a meeting, to the water cooler, with the boss, until you finally return back to home base, which is revealed as an end of the row basement cubicle.

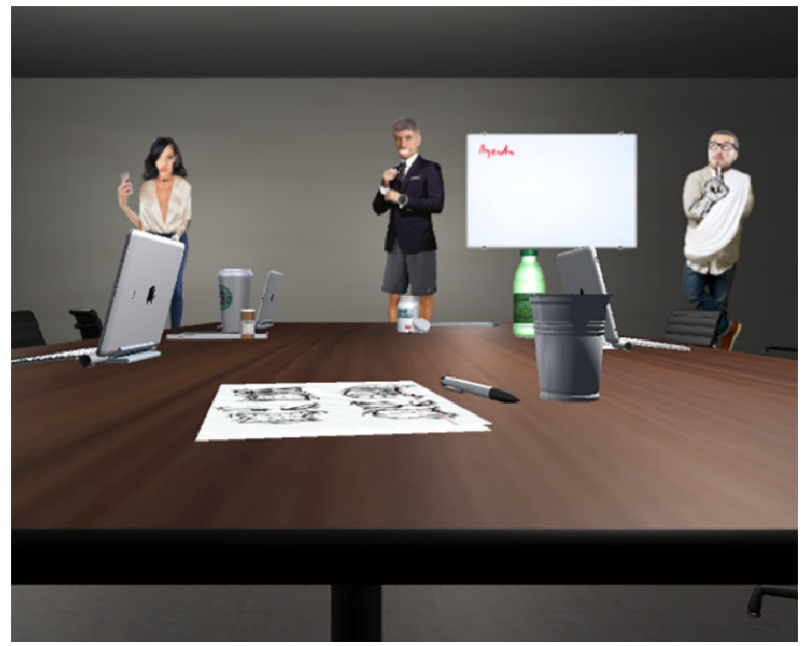

Figure 2: Meeting with Archetypical Colleagues

Every interruption is another level, and each character the user encounters reflects a different type modern day archetype. The nature of the interactions is itself as a form of computer mediated communication. The Artiste, for example, shares her unique creations and waits expectantly for your approbation. The Psychotic, rather, demands that you comply with his standards of safe behaviour in a world of online spies. His seeming insanity becomes a touchstone for realistic assessment of the world in which you operate. 


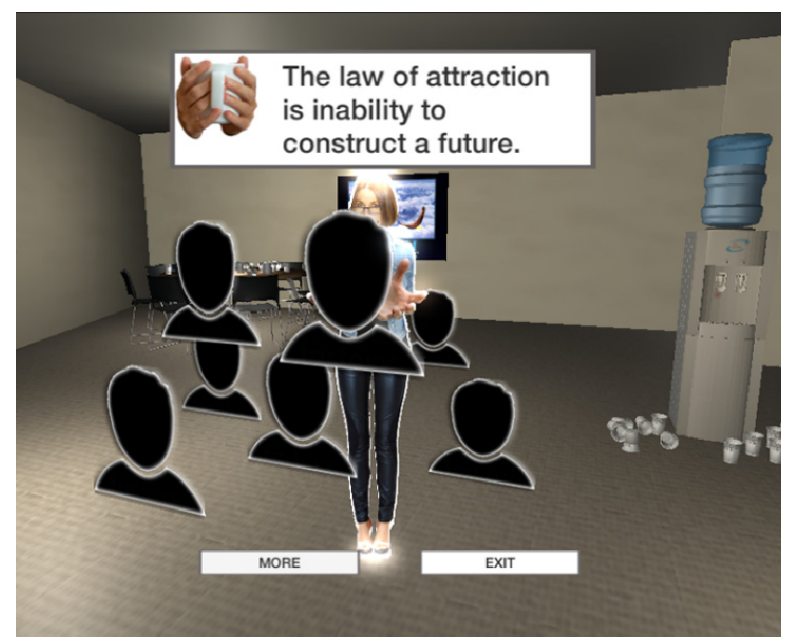

Figure 3: The Bombast shares her wisdom

These archetypical figures also include the Egoist, the Bombast, the Sophist, the Celebrity, the Dominator and more.

The Egoist, for example, shows up as the Doctor, whose finely tuned scientific instruments resemble Buzzfeed quizzes as much as they do the Jungian word associations by which they were inspired. How the user engages with each of these characters adds to the system's cumulative judgement of overall workplace fitness. Scrolling text relevant to each level, along with an office decor that changes depending on previous interactions, also hint at the ongoing data-gathering taking place within the system.

During the endgame, the archetypal figures with which the user has engaged recombine into a modern day tarot. This is presented alongside a "Success Index" derived based on gameplay choices and behaviours, and which transforms the popular OCEAN Five Factors personality test, which measures Openness, Conscientiousness, Extraversion, Agreeableness, and Neuroticism, but re-imagining them as Gullibility, Grinding, Gladhanding, Subjugation, and Internalisation.

As the game progress, the user comes to learn that nothing within the workplace remains outside the evaluatory system, and that even the most trivial of engagements has an effect upon the user's ultimate rating. For example, when the user interacts with the Celebrity, the responses are limited to various degrees of affirmation. Anything less than fulsome praise will come back in the form of a negative "Collusion" score. Tolerating the Bombast's auto-generated aphorisms, or succumbing the Charismatic's gaslighting techniques increment the Gladhanding and Internalisation scores, respectively. Merely enduring the Dominator's self-aggrandisement yields positive rewards on the Grinding index.

\section{RESULTS}

Because the game itself is delivered on a computer as a work of new media art, Dupes ultimately also complicates our ever-more-entangled relationships with computer mediated communication by making it unclear whether or not the user's own gameplay is being re-appropriated into the system. We place the user in the uneasy position of not being sure whether they themselves are in on the joke as individuals playing a cynical game, or if their engagement in our project is itself being logged and judged through their game interactions for the purpose (perhaps) of our future projects as the game's creators.

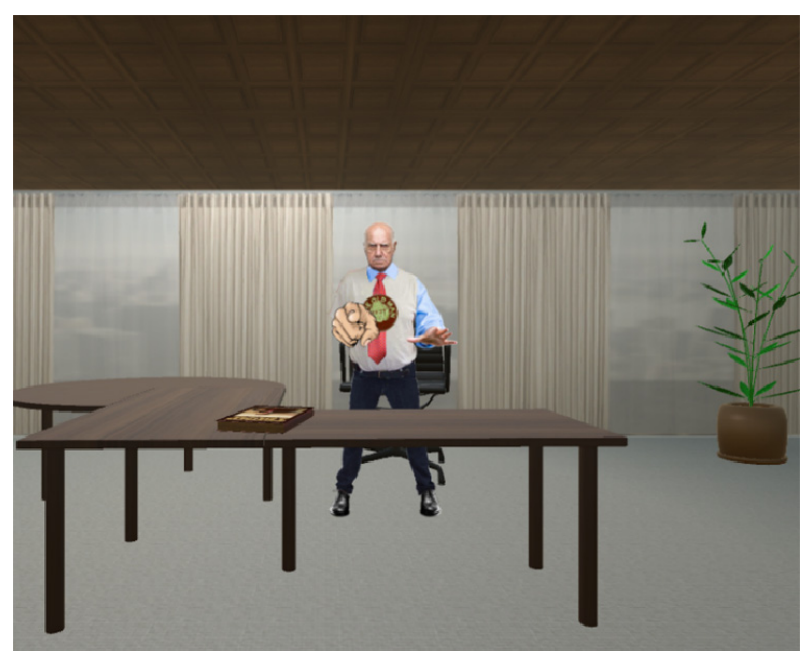

Figure 4: Encountering the Boss

As our earlier experiences with the Pyschasthenia series suggest, this suspicion and ambiguity of purpose create a paranoid worry that contributes to the overall effect of the installation. The subject position of the user as a Dupe puts him or her back into the endless regression of surveillance and recuperation, making us as the game's creators also inherently complicit with the system.

\section{REFERENCES}

Rudinsky, J. and Szabo, V. (2017) Psychasthenia Studio. http://psychasthenia-studio.com (accessed 22 March 2017). 